\title{
Magnetic Field Effects on Electrochemical Property of Self-Assembled Monolayers With Charge-Transfer Groups
}

\author{
Noriyuki Yoshimoto, Kaoru Ogawa, and Satoshi Ogawa
}

\begin{abstract}
Novel self-organization molecules, $\boldsymbol{n}$-mercapto-alkyl benzo-annelated tetrathiafulvalenes (BTTF), have been synthesized. Exposure of polycrystalline $\mathrm{Au}$ and oriented crystalline $\mathrm{Au}$ (111) surfaces to organic solution of the novel BTTF derivatives in high magnetic field results in the binding of the derivatives to Au and the formation of highly oriented self-assembled monolayers (SAMs) with redox active tetrathia-fulvalene (TTF) unit. The electrochemical properties were confirmed in the monolayer state. A comparison between the SAMs on polycrystalline Au having a rough surface and on oriented crystalline Au (111) having a flat surface suggested that the well-oriented alkyl-chain layers in the SAMs could be a blockade for the electron transfer between the TTF unit and the Au substrate.
\end{abstract}

Index Terms-Electron transfer, oriented Au (111) surface, selfassembled monolayers, superconducting magnet.

\section{INTRODUCTIONS}

$\mathbf{S}$ URFACE modification with self-assembled monolayers (SAMs) is of fundamental technological interest because of their potential in the molecular design of interfacial structure and application to the two-dimensional molecular arrays. In practice, SAMs adsorbed on well-defined substrates serve as electrode modification, protein recognition and adsorption, patterning of surfaces, controlled introduction of surface defects, and controlled wetting [1]. Organosulfur monolayers that serve to redox active species to a gold electrode present a unique opportunity to investigate a number of phenomena, including electron transfer rates and mechanisms. The electrochemical characteristics for SAMs containing redox couples such as TTF have been reported [2]-[4]. In their reports, the investigation of distance dependence of long-range electron transfer kinetics was based on the varying thickness of a thiol-functionalized SAM as a spacer between the electrode and a redox center. However, although simple alkyl thiol assemblies were readily adsorbed to form well-defined SAMs, [5], [6] the alkyl thiols with bulky head-group such as TTF unit were deviated from

Manuscript received October 20,2003. This work was supported in part by Japan Science and Technology Corporation under the Joint-Research Project for Regional Intensive in the Iwate Prefecture on "Development of practical applications of magnetic field technology for use in the region and everyday living,"

N. Yoshimoto and S. Ogawa are with the Faculty of Engineering, Iwate University, Morioka 020-8551, Japan (e-mail: yoshimoto@iwate-u.ac.jp; ogawa@iwate-u.ac.jp)

$\mathrm{K}$. Ogawa is with the Iwate Industrial Promotion Center, Advanced Science and Technology Institute of Iwate, Morioka 020-0852, Japan (e-mail kaoru@iwate-u.ac.jp)

Digital Object Identifier 10.1109/TASC.2004.830729 well-arrange structure by the size mismatch between the anchor (sulfur atom) and the head (TTF unit) group.

As part of our interest in this area, we have been searching for approaches to study the fundamental chemical and physical interactions that control the formation, structure, and reactivity of chemically modified surfaces. Recently, in the field of material science and technology it has been found that a molecular orientation in materials can be controlled by imposition of a high magnetic field. We attempted to utilize a magnetic field for formation of highly ordered SAMs with bulky electronically active TTF unit. In this paper, we show the magnetic effects on the redox behavior of our well-defined SAMs bound to charge transfer TTF group.

\section{EXPERIMENTAL}

\section{A. Syntheses of Self-Organization Molecules}

All new molecules were synthesized by chemical methods as shown in Fig. 1. All solvents used in the reactions were purified by the general methods. Melting and decomposition points were determined on a Mel-Temp capillary tube apparatus. ${ }^{1} \mathrm{H}$ $(400 \mathrm{MHz})$ and ${ }^{13} \mathrm{C}(101 \mathrm{MHz}) \mathrm{NMR}$ spectra were measured on a Bruker $\mathrm{AC}-400$ spectrometer using $\mathrm{CDCl}_{3}$ as a solvent with $\mathrm{Mc}_{4} \mathrm{Si}$ as the internal standard. IR (Infrared) spectra were recorded on a JASCO FT-7300 spectrometer. Mass spectra were determined on a Hitachi M-2000 spectrometer operating at $70 \mathrm{eV}$ in the EI mode. Elemental analyzes were carried out by a Yanagimoto MT-5 CHN recorder. Silica-gel column chromatography for purification of desired molecules was performed with a Wakogel C-200.

\section{B. Self-Assembled Monolayer Preparation}

Polycrystalline Au substrates are commercially available. All oriented $\mathrm{Au}(111)$ substrates were prepared by vacuum evaporation at $1.0 \times 10^{-8} \sim 10^{-9}$ Torr onto cleaved mica (001) $(20 \mathrm{~mm}$ $\times 20 \mathrm{~mm}$ ) that previously pre-baked at $400^{\circ} \mathrm{C}$ for $24 \mathrm{~h}$ under reduced pressure $\left(1.0 \times 10^{-8} \sim 10^{-9}\right.$ Torr $)$. The substrates were coated with $500 \AA$ gold films (substrate temperature, $400^{\circ} \mathrm{C}$; deposition rate, $1.0 \AA / \mathrm{min}$ ) after annealing at $450^{\circ} \mathrm{C}$ under reduced pressure $\left(1.0 \times 10^{-8} \sim 10^{-9}\right.$ Torr $)$.

Monolayer films were typically prepared by immersing a substrate (polycrystalline $\mathrm{Au}$ or oriented crystalline $\mathrm{Au}(111)$ ) into a $1.0 \mathrm{mM}$ dichloromethane solution of the precursor for $24 \mathrm{~h}$ (Fig. 2) under the high magnetic field of $10 \mathrm{~T}$ produced by a super conducting magnet JMTD-10 T 100 M (Japan Magnet

$1051-8223 / 04 \$ 20.00$ (O) 2004 IEEE 


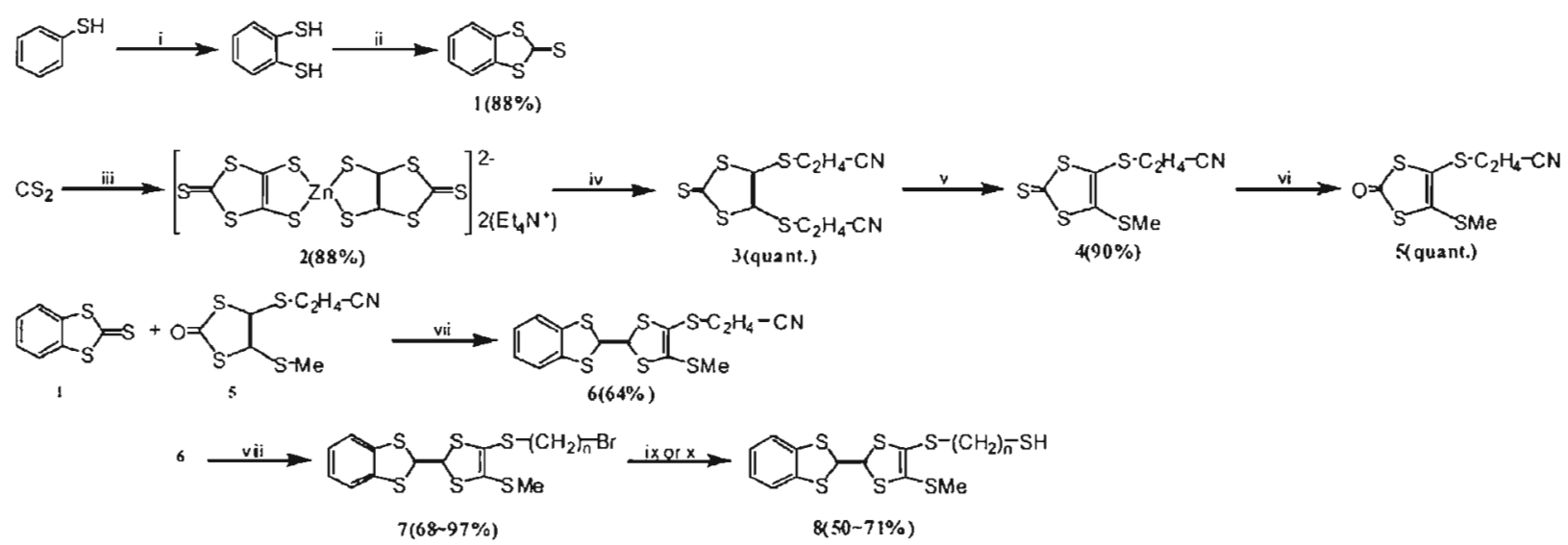

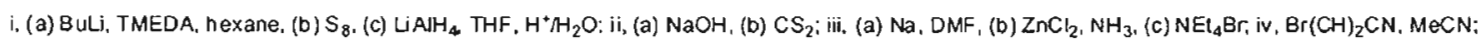

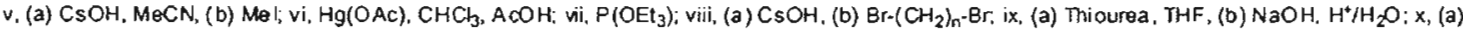
$\mathrm{KSCOCH}_{3}, \mathrm{OMF}$, (b) $\mathrm{H}^{*} \mathrm{H}_{2} \mathrm{O}$. ETOH

Fig. 1. Synthesis of self-organization molecules
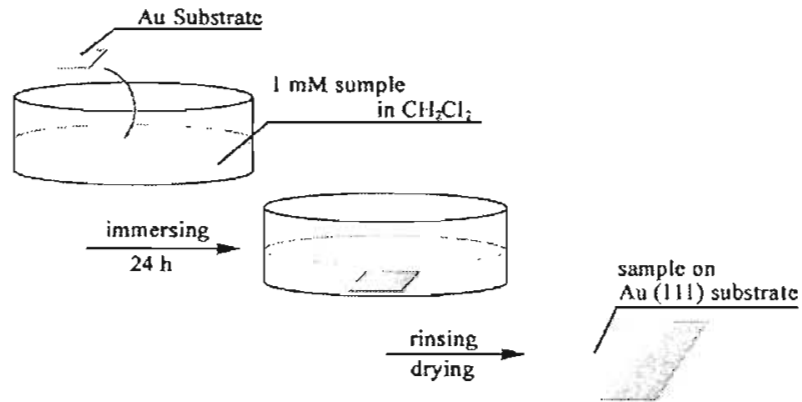

Fig. 2. Preparation of SAMs.

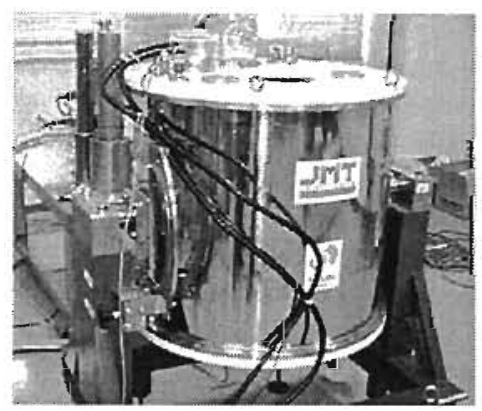

Fig. 3. Superconducting magnet.

Technology Ltd., $100 \mathrm{~mm} \phi$ ) (Fig. 3). The directions of the magnetic field were in parallel $(/ /)$ and perpendicular $(\perp)$ to the direction of the gold surface. The substrate was then removed from solution, sequentially rinsed with fresh dichloromethane, and dried with flowing nitrogen prior to analysis.

\section{Characterization}

Cyclic voltammetry was performed with a Cypress System CS-1090 galvamostat/potentiostat. The gold substrates were mounted in a conventional three-electrode cell with an exposed area $19.6 \mathrm{~mm}^{2}$ (5.0 $\left.\mathrm{mm} \phi\right)$ ). as shown in Fig. 4. Redox potentials of the SAMs were measured with respect to an $\mathrm{Ag} / 0.01 \mathrm{M}$

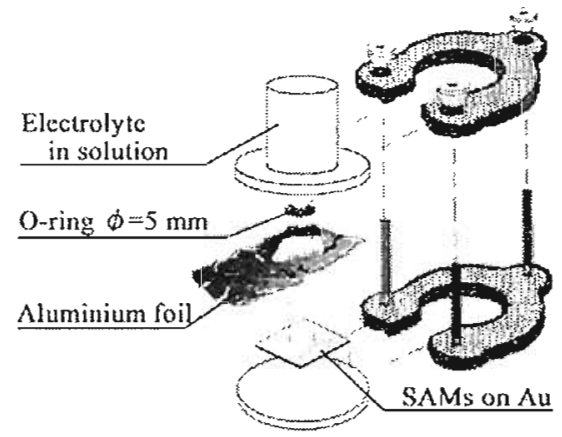

Fig. 4. CV measurement cell.

$\mathrm{AgNO}_{3}$ reference electrode. The measurements were carried out in acetonitrile solution containing $0.1 \mathrm{M} \mathrm{Bu}_{4} \mathrm{NClO}_{4}$ as a supporting electrolyte. Reductive eliminations of the SAMs were measured with respect to an $\mathrm{Ag} / \mathrm{AgCl}$ (saturated $\mathrm{KCl}$ ) reference electrode. The measurements were carried out in water solution containing $0.1 \mathrm{M} \mathrm{KOH}$ as a supporting electrolyte.

Polarization modulation infrared reflection absorption spectroscopy (PM-IRRAS) data were obtained using a Nicolet NEXUS-IR 670 Fourier transform spectrometer equipped with a liquid nitrogen-cooled mercury-cadmium-telluride (MCT) detector and a Hinds Instruments PEM-90 photoelastic modulator operating at $37 \mathrm{kHz}$. The polarized light was reflected from the sample at an angle of incidence of $83^{\circ}$. The spectra were collected 300 scans at a spectral resolution of $4 \mathrm{~cm}^{-1}$.

\section{RESULTS AND DISCUSSION}

Fig. 5 shows the typical cyclic voltammograms for the spontaneously adsorbed BTTF $(n=8)$ self-assembled monolayers on both polycrystalline Au and oriented crystalline Au (111) without high magnetic field. As expected for the voltammetric behavior of the surface-confined redox center, the redox peak current is linearly proportional to scan rate up to $500 \mathrm{mV} / \mathrm{s}$ (used in this study), and the ratio of $I_{\mathrm{pat}}$ to $I_{\mathrm{pc}}$ at given scan rate is 
i ) SAMs / polycrystalline

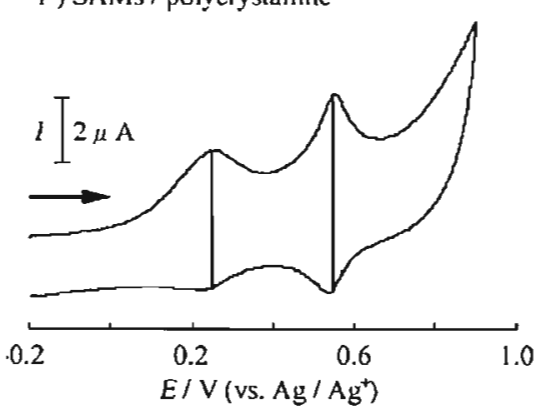

ii ) SAMs / oriented crystalline

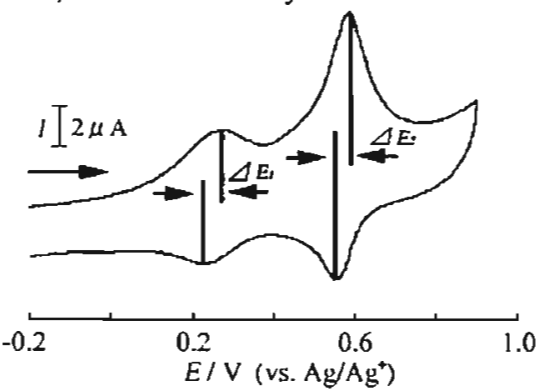

Fig. 5. Cyclic yoltammograms of BTTF SAMs. i) SAMs/polycrystalline: ii) SAMs/oriented crystalline.

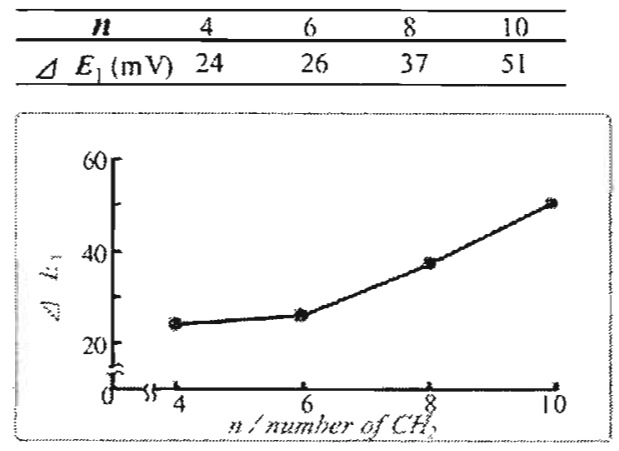

Fig. 6. $\Delta E_{1}$ values of $\operatorname{BTTF}(n=4 \sim 10)$.

very close to unity for all of these BTTF monolayers. This indicates that the electroactive BTTF monolayers were prepared in reproducible form. In each voltammogram, two pairs of redox peaks observed due to the oxidation and reduction of BTTF which follows two-step two-electron transfer reaction mechanisms. For the BTTF SAMs on polycrystalline Au, a symmetrical reversible redox waves were observed and the peak-to-peak separation $\left(\Delta \mathrm{E}_{\mathrm{p} 1}\right.$ and $\left.\Delta \mathrm{E}_{\mathrm{p} 2}\right)$ values, although not zero, were typically very small, suggesting a rapid electron transfer reaction kinetics in the condition. On the other hand, the significantly large $\Delta E_{p}$ values were observed for BTTF SAMs on oriented crystalline $\mathrm{Au}$ (111). As can be seen in Fig. 6, the value of $\Delta E_{p 1}$ measured at the same scan rate remarkably increases as the number of methylenes in the alkyl chain spacer increase ( $n=4,6,8$, and 10). When the alkyl chain length becomes longer, the electron transfer from the redox center to the electrode is forced to proceed at a large distance, slowing the overall electron-transfer rate.

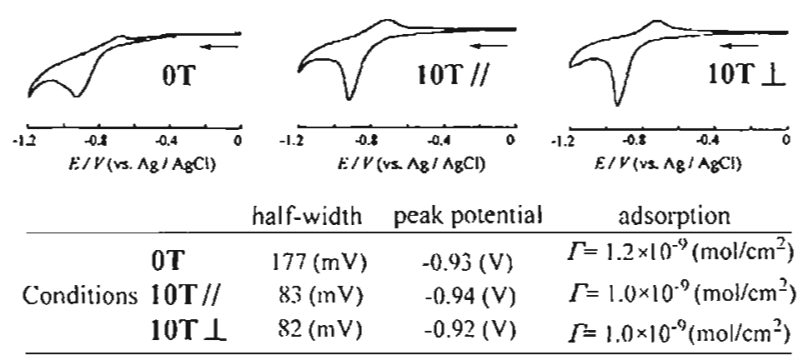

Fig. 7. Cathodic electrochemical properties of BTTF.

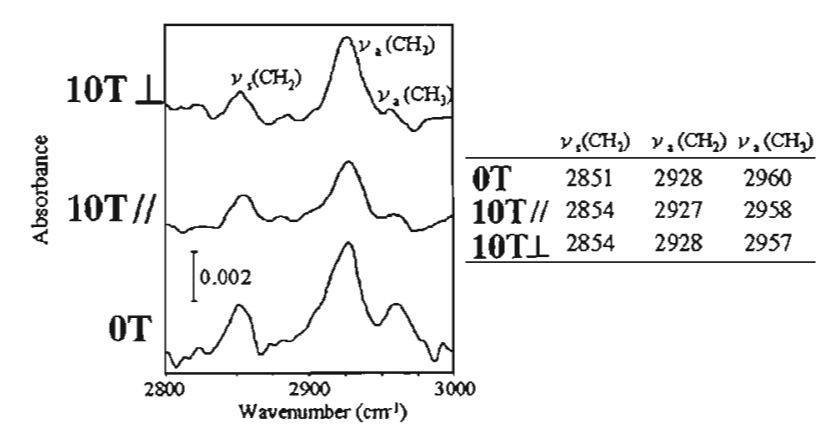

Fig. 8. Surface infrared spectra of BTTF SAMs.

As further advances for formation of high-quality BTTF SAMs on oriented crystalline Au (111) surface, we prepared BTTF SAMs $(\mathrm{n}=8)$ in bigh magnetic field $(10 \mathrm{~T})$. In order to begin to examine the effect of high magnetic field. we have exploited cathodic electrochemical measurement (Fig. 7).

For a BTTF-covered Au (111) surface desorption of the monolayers takes place at current peak $\left(E_{p x}\right)$ located at -0.93 $\mathrm{V}(0 \mathrm{~T}),-0.94 \mathrm{~V}(10 \mathrm{~T} / /),-0.92 \mathrm{~V}(10 \mathrm{~T} \perp)$ (all scan rate, $100 \mathrm{mV} / \mathrm{s})$, respectively. The results indicate that one-electron transfer for desorption of a thiol from Au latice takes place. Although the $\mathrm{E}_{\mathrm{pc}}$ values of BTTF SAMs in high magnetic field were close to that value without using high magnetic field, the half-width of the peak potentials of the SAMs produced in high magnetic field were narrow compared with that obtained without using high magnetic field. If balf-width of the peak narrow, the monolayer possesses the higher ordering. For the low ordering monolayer, the half-wave becomes broader. On the other hand, the surface coverage $(\Gamma)$ values of BTTF SAMs were calculated from the integration of reduction peak area. Unexpectedly, the quantities of the monolayers were nearly equal in all conditions. The results described above imply that packing structure of the monolayers on the oriented crystalline Au (111) becomes more ordered with growing micro domains in high magnetic field. Fig. 8 shows the $\mathrm{C}-\mathrm{H}$ stretching region of the PM-IRRAS spectra obtained for the SAMs derived from BTTF $(n=8)$. In evaluating the data from the PM-IRRAS measurements, we focus our attention on the frequency of the methylene antisymmetric $\mathrm{C}-\mathrm{H}$ stretch $\left(v_{a}^{\mathrm{CH}} 2\right)$ and methyl antisymmetric $\mathrm{C}-\mathrm{H}$ stretch $\left(v_{\mathrm{a}}^{\mathrm{CH} 3}\right)$, because these bands are sensitive to the degree of ordered of the films; the lower the values, the greater the crystallinity of the SAMs. As with the cyclic voltammmetric half-width data shown in Fig. 7, 
antisymmetric $\mathrm{C}-\mathrm{H}$ band, especially shift of $v_{\mathrm{a}}^{\mathrm{CH} 3}$, suggested well-ordered SAMs could be formed in high magnetic field.

\section{CONCLUSION}

A series of specifically designed BTTF molecules (the number of methylene in alkyl chain; $C=4,6,8$, and 10) were used to prepared SAMs on oriented crystalline Au (111) in which the well-oriented alkyl-chain layers in the SAMs could be a blockade for the electron transfer between the TTF unit and the Au substrate. Furthermore, high-quality BTTF SAMs on oriented crystalline Au (111) surface have been achieved by the use of superconducting magnet. High magnetic fields are effective for the formation of two-dimensional self-assembled monolayers by maganetohydrodynamic effect and/or magnetic anisotropy effect to diamagnetic organic molecules. Further studies on the mechanism for formation of BTTF SAMs on oriented crystalline $\mathrm{Au}$ (111) in high magnetic field are presently underway.

\section{REFERENCES}

[1] A. Ulman, An Introduction to Organic Films: From Langmuir-Blodgett 1o Self-Assembly. San Diego: Academic Press, 1991

[2] C. M. Yip and M. D. Ward, "Self-assembled monolayers with charge-transfer groups: $n$-mercaptoalkyl tetrathiafulvalenecarboxylate on gold." Langmuir, vol. 10, pp. 549-556, 1994.

[3] R. Yuge, A. Miyazaki. T. Enoki, E. Ito, F. Nakamura, and M. Hara, "Characterization and electronic properties of TTF SAMs on Au (111)," Mol. Cryst. and Liq. Cryst., vol. 370, pp. 273-276, 2001

[4] N. Yoshimoto, K. Ogawa, Y. Hiroi, and S. Ogawa, Synthesis and electrochemical property of self-assembled monolayers with charge transfer group, in TMS Annual Report, Supplement II, pp. 245-246, 2001.

[5] M. D. Poter, T. B. Bright, D. L. Allara, and C. D. E. Chidsey, "Spontaneously organized molecular assemblies. 4. Structural characterization of $n$-alkyl thiol monolayers on gold by optical ellipsometry, infrared spectroscopy, and electrochemistry," J. Am. Chem. Soc., vol. 109, pp. $3559-3568,1987$

[6] G. E. Poirier, W. P. Fitts, and J. M. White, "Two-dimensional phase diagram of decanethiol on Au (11 1)," Langmuir, vol. 17, pp. 1176-1183, 2001 